\title{
Green Lightpath Provisioning in Transparent WDM Networks: Pros and Cons
}

\author{
Pawel Wiatr, Paolo Monti, Lena Wosinska \\ Next Generation Optical Networking (Negonet) Group \\ School of Information and Communication Technology (ICT) \\ Royal Institute of Technology (KTH) \\ Isafjordsgatan 22, 16440 Kista, Sweden
}

\begin{abstract}
Despite their intrinsic power efficiency, transparent wavelength division multiplexing (WDM) networks have additional potentials to save energy, and have attracted the interest of researchers with many power-aware $(\mathrm{PA})$ routing and wavelength assignment (RWA) solutions being proposed in the literature. These approaches seem to have, however, an adverse effect on network blocking probability performance. This paper aims at providing a different insight to the PA-RWA problem. The idea is based on the intuition that power minimization and resource blocking can be jointly treated. The proposed approach, called Weighted Power-Aware RWA (WPA-RWA), has been tested using a continental core network and the performance evaluation confirms the presence of a trade-off between power saving and blocking probability.
\end{abstract}

\section{INTRODUCTION}

Optical networks are a power efficient transport option able to play an important role in reducing the power consumption in the ICT sector. The main benefit of optical networks derives from their ability to transparently, i.e., all-optically, transmit data from source to destination, thus avoiding power hungry optical-electrical-optical (O-E-O) conversion [1]. Recently, several solutions have been proposed in the literature to further improve the power efficiency of transparent WDM networks. These approaches address the power consumption in both the network design [2] and the path provisioning phase [1]. Among these methods, solutions limiting the number of active network elements during normal network operations attracted a lot of attention.

These solutions are currently advocated in both static and dynamic provisioning scenarios. In a static paradigm [2]-[4] these methods are used to re-provision traffic in previously deployed network, where the objective is to lower the total network power consumption by having a minimum number of active elements. In a dynamic scenario [5] links with traffic below a certain threshold may be set to an off/sleep state and their traffic rerouted. Regardless of the provisioning scenario, the focus of these solutions is on power minimization only. Currently, in the literature little or no attention is paid to the effect that solutions tailored only to lower the power consumption has on other network metrics. For example, a pure power minimization provisioning strategy may have an adverse impact on the length of the routed lightpaths, i.e., in order to avoid to unnecessarily power on network elements, the provisioned paths are, on average, longer than the one found with traditional shortest path solutions. This is in contradiction with the goal of classical routing and wavelength assignment (RWA) algorithms that tend to minimize the resource usage within the network, in order to minimize the blocking probability.

This paper addresses this problem by investigating the impact that power minimization has on the overall network performance. With this purpose in mind, a non-conventional solution to the power-aware RWA problem is presented. This approach, referred to as Weighted Power-Aware RWA (WPARWA), is based on the intuition that in some cases relaxing the power minimization constraint can have beneficial effects on the overall network performance, i.e., it can contribute to the reduction of resource fragmentation in the network and lower the blocking probability. The proposed algorithm is very promising and leverages on a cost function that weights the power status of network elements versus information about the wavelength usage. Performance results confirm the intuition that an approach that accounts only for the minimization of the network power consumption might not always be the best choice, especially in a resource limited network scenarios where network blocking cannot be ignored.

\section{A WEIGHTED POWER -AWARE ROUTING AND WAVELENGTH ASSIGNMENT STRATEGY}

This section first presents the network power model, and then it describes the proposed Weighted Power-Aware RWA (WPA-RWA) strategy.

This study assumes a WDM network where nodes are equipped with optical cross-connects (OXC) and fiber links are equipped with one or more in-line optical amplifier, depending on the fiber link length. Connection requests are provisioned all-optically from source to destination, i.e., no opticalelectrical-optical (O-E-O) conversion takes place at intermediate nodes. Based on these assumptions, the power required to provision one connection request is equal to the sum of: (i) the power consumption of the transceiver, (ii) the power needed to optically switch the signal at intermediate nodes, i.e., OXCs, and (iii) the power consumed by the in-line optical amplifiers along each fiber link in the path. The overall network power consumption, at any given time, is the sum of the power consumed by all the connection requests currently 
provisioned.

The proposed WPA-RWA strategy is based on a modified version of the k-shortest path algorithm [6], and it works as follows. For each connection request arriving at the network edge, up to $k$ candidate paths are computed. The algorithm accounts for the current resource usage (i.e., wavelengths) in the network, where fiber links without available wavelengths are temporarily deleted from the network topology. When computing each candidate path, each fiber link $l$ in the network is assigned a weight $\left(C_{l}\right)$ as follows:

$$
C_{l}=\left\{\begin{array}{l}
\alpha \cdot P_{\text {link }, l}, \text { fiber link } l \text { in use } \\
P_{\text {link }, l}, \text { fiber link } l \text { not in use }
\end{array}\right.
$$

where $P_{\text {link, }, l}$ represents the power necessary to operate the inline power amplifier(s) along fiber link $l$, and $\alpha$ is a weighting factor with values between 0 and 1 . Note that with values of $\alpha$ close to 0 WPA-RWA behaves as a pure power minimization approach, while for values of $\alpha$ close to 1 WPA-RWA tends to provision connection requests along shorter routes.

During the first phase, a set of $k$ paths are sorted in ascending order based on their power consumption. At this point, the first computed path, among the $k$, able to accommodate a continuous wavelength, is chosen to provision the connection request. If, among the $k$ computed paths, no continuous wavelength can be found, or no candidate path can be computed at all, the connection request is blocked.

\section{RESULTS}

This evaluates the impact that the WPA-RWA strategy has on the blocking probability and the average fiber link utilization. The presented results are obtained considering the COST 239 network topology with 11 nodes and 26 bidirectional fiber links [7]. It is assumed that each fiber carries a maximum of sixteen wavelengths and no wavelength conversion capability is considered in the network. Source destination pairs for the incoming connection requests are uniformly distributed among the networks nodes. Furthermore, it is assumed that the connection requests arrive according to the Poisson distribution with parameter $\lambda$, while the service time for each connection request is exponentially distributed with a mean holding time defined by $1 / \mu$. The power consumption assumptions for the optical components are the following. In-line optical amplifiers (EDFAs), placed every 80 $\mathrm{km}$, consume $12 \mathrm{~W}$. A transceiver consumes $7 \mathrm{~W}$ (for a transmission speed of $10 \mathrm{Gbps}$ ), and an OXC $6.4 \mathrm{~W}$. The values are obtained from available data sheets [8]-[10]. A series of values for the power-weighting factor $\alpha$ are considered, starting from 1 down to values of $\alpha$ close to 0 . When $\alpha=1$ WPA-RWA tries to reduce the average length of a provisioned connection request, while when the value of $\alpha$ is close to zero the objective of WPA-RWA is the minimization of the power consumed by each provisioned connection request. In this study no more than $k=3$ candidate paths are computed for each connection request, while wavelengths are assigned using a First Fit approach.

Figures 1 and 2 show the values of the network blocking probability, and the average power saved per request, respectively. Both metrics are presented as a function of the network load. The average power saved per request is computed as the difference between the total network power consumption obtained when $\alpha=1$ and the total network power consumption for any other given value of $\alpha$.

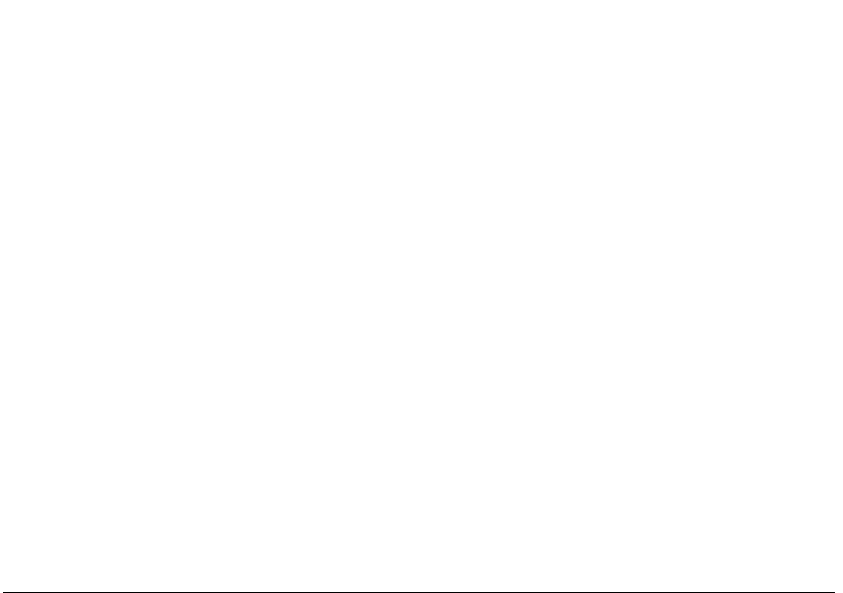

Figure 1. Network blocking probability vs. the network load.

The figures show that considerable power savings (up to $50 \%$ ) are achievable, but at the expense of a relevant increase in the network blocking probability. This confirms the earlier intuition that an approach that accounts only for the minimization of the network power consumption may not always be the best choice. The figures also show the possible tradeoff results between these two metrics, should an operator be willing to compromise on the total network power expenditure. For example with values of $\alpha$ between 0.66 and 1 , there is no significant impact on the blocking probability, but the power saved per request is still significant, e.g., between $30 \%$ and $15 \%$ for low and medium traffic conditions.

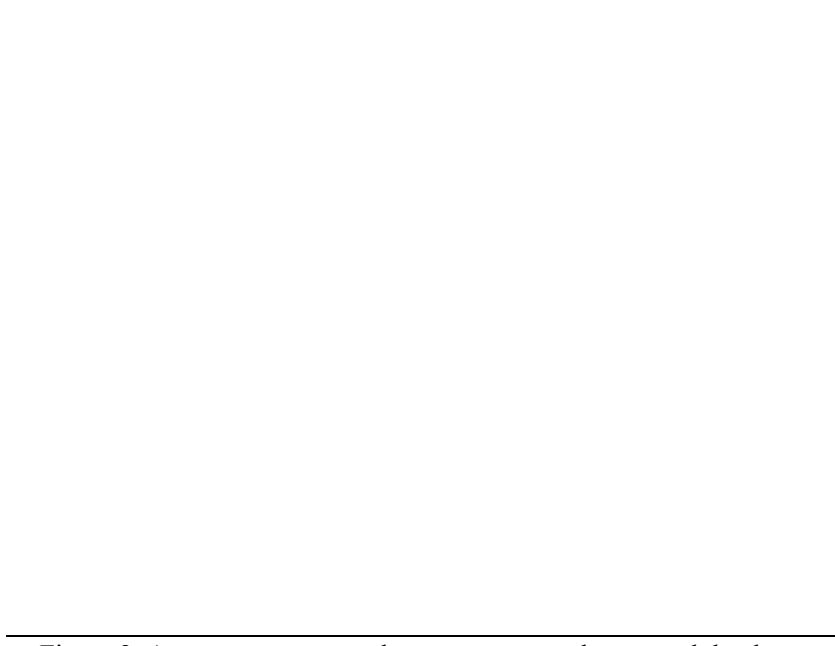

Figure 2. Average power saved per requests vs. the network load. 
Figure 3 shows the percentage of fiber link used in the network as a function of the network load. As expected the power saving figures presented previously are the result of an efficient utilization of network elements that are already powered on.

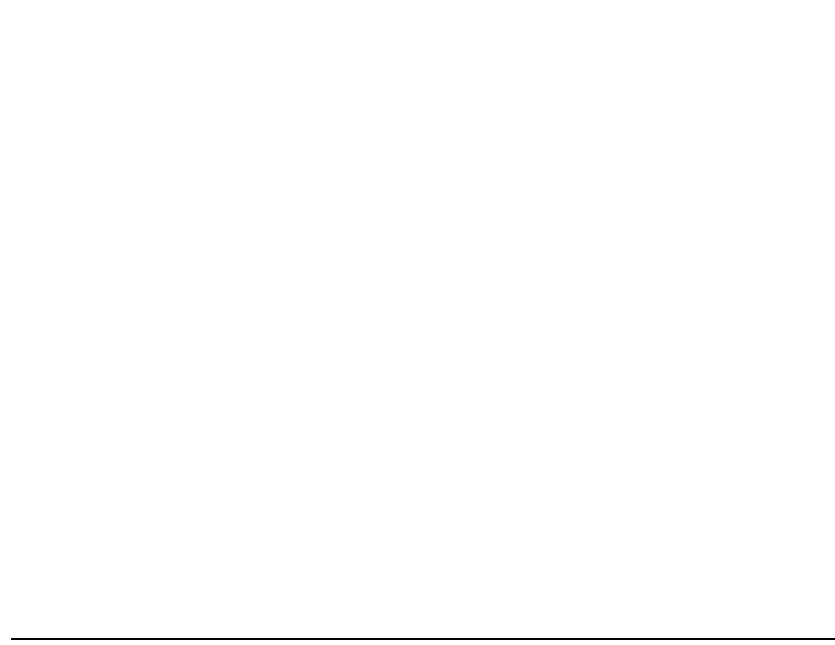

Figure 3. Average number of link use in the network vs. the network load.

When the WPA-RWA strategy focuses solely on the minimization of the length of the provisioned paths the fiber links utilization raises very quickly with an increased value of the load. This is reasonable since with value of $\alpha$ close to one WPA-RWA does not consider the power status of the network elements, and cares only about guaranteeing the shortest available path to the provisioned connection requests. This is true already at low traffic conditions where more than $75 \%$ of the network fiber links in the network are powered on accommodate the traffic. When the value of $\alpha$ is close to 0 the fiber link utilization increases almost linearly with the load. This translates into more fiber links (and consequently in-line amplifiers) that are not used in the network.

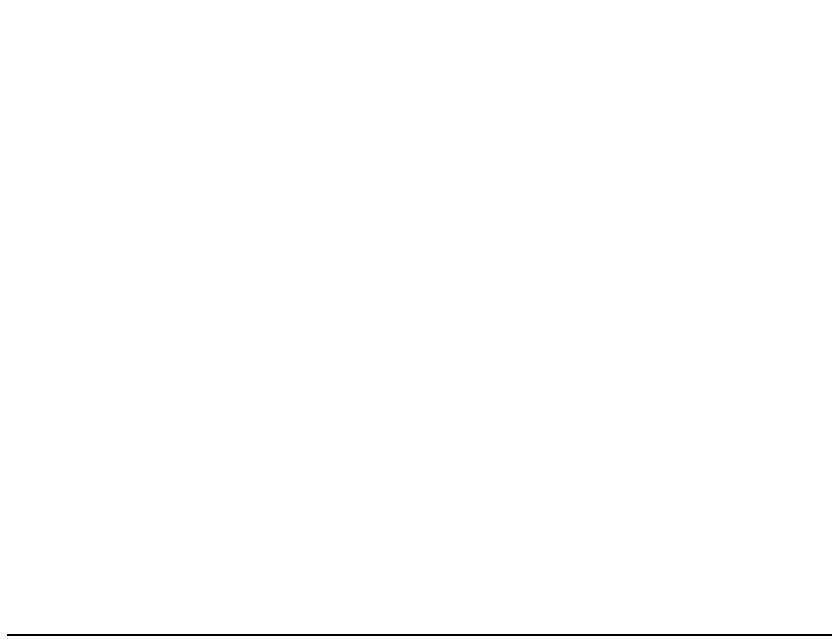

Figure 4. Average number of wavelength used on a fiber link vs. the network load.
This difference in the utilization of the fiber links has also an immediate impact on the fiber link occupancy, as it is shown in Figure 4. Fiber links are on average less utilized when $\alpha$ is close to 1 . As a result the traffic is spread more evenly over the network and the chance to have bottlenecked fiber links is decreased. This is not true anymore when $\alpha$ gets closer to 0 , i.e., when WPA-RWA tends to provision as much as possible connection requests using network elements already used. Consequently, fiber links are on average more utilized, with a higher chance to find bottlenecks.

\section{CONCLUSIONS}

Power efficiency and network blocking probability are two conflicting objective, i.e., a path resulting from a pure poweraware provisioning may be too long thus increasing the network resources fragmentation level. Consequently, the network blocking probability may also increase. This can be avoided by jointly considering power minimization and resource blocking in a single cost function as advocated by the Weighted Power-Aware Routing and Wavelength Assignment (WPA-RWA) strategy proposed in this paper. Simulation results confirm the advantage of the proposed power-aware solution in terms of both power and resource usage efficiency and suggest that a "binary" approach using only poweredon/powered-off information can lead to a significant degradation of network performance.

\section{ACKNOWLEDGMENT}

The work described in this paper was carried out with the support of the BONE-project ("Building the Future Optical Network in Europe"), a Network of Excellence funded by the European Commission through the 7th ICT-Framework Programme.

\section{REFERENCES}

[1] Y. Zhang, P. Chowdhury, M. Tornatore, and B. Mukherjee, "Energy Efficiency in Telecom Optical Networks," IEEE Com. Surveys \& Tutorials, vol. 2, no. 4, 2009.

[2] Y. Wu, L. Chiaraviglio, M. Mellia, and F. Neri, "Power-Aware Routing and Wavelength Assignment in Optical Networks," in Proc. of ECOC, 2009.

[3] A. Muhammad, P. Monti, I. Cerutti, L. Wosinska, P. Castoldi, and A. Tzanakaki, "Energy-Efficient WDM Network Planning with Dedicated Protection Resources in Sleep Mode," in Proc. of GLOBECOM, 2010.

[4] C. Cavdar, F. Buzluca, and L. Wosinska, "Energy-Efficient Design of Survivable WDM Networks with Shared Backup," in Proc. of GLOBECOM, 2010.

[5] I. Cerutti, N. Sambo, and P. Castoldi, "Distributed Support of Link Sleep Mode for Energy Efficient GMPLS Networks," in Proc. of ECOC, 2010.

[6] E.Q.V. Martins, and M.M.B. Pascoal, "A New Implementation of Yen's Ranking Loopless Paths Algorithm”, 4OR, vol. 1, no. 2, 2003.

[7] P. Batchelor et al., "Study on the Implementation of Optical Transparent Transport Networks in the European Environment-Results of the Research Project COST 239", Photonic Network Communications, vol. 2, no. 1, 2000.

[8] Light "10-GigE Reading, Transponders: Update", http://www.lightreading.com/document.asp? site=lightreading\& doc $i d=43536$ \&page number $=1$, Nov. 2003.

[9] G. Shen, and R.S. Tucker, "Energy-Minimized Design for IP Over WDM Networks", IEEE/OSA J OCN, vol. 1, no. 1, 2009.

[10] S. Aleksic, "Analysis of Power Consumption in Future High-Capacity Network Nodes", IEEE/OSA JOCN, vol. 1, no. 3, 2009. 